\title{
Joint IA and SFBC Macrocells and Small-Cells Coexistence under Minor Information Exchange
}

\author{
Syed Saqlain Ali, Daniel Castanheira, Adão Silva, and Atílio Gameiro
}

Instituto de Telecomunicações, University Campus, 3810-193 Aveiro, Portugal

Correspondence should be addressed to Syed Saqlain Ali; syedsaqlain@av.it.pt

Received 8 October 2015; Accepted 20 January 2016

Academic Editor: Yuh-Shyan Chen

Copyright (c) 2016 Syed Saqlain Ali et al. This is an open access article distributed under the Creative Commons Attribution License, which permits unrestricted use, distribution, and reproduction in any medium, provided the original work is properly cited.

\begin{abstract}
The deployment of small-cells within the boundaries of a macrocell is considered to be an effective solution to cope with the current trend of higher data rates and improved system capacity. In the current heterogeneous configuration with the mass deployment of small-cells, it is preferred that these two cell types will coexist over the same spectrum, because acquiring additional spectrum licenses for small-cells is difficult and expensive. However, the coexistence leads to cross-tier/intersystem interference. In this context, this contribution investigates interference alignment (IA) methods in order to mitigate the interference of macrocell base station towards the small-cell user terminals. More specifically, we design a diversity-oriented interference alignment scheme with space-frequency block codes (SFBCs). The main motivation for joint interference alignment with SFBC is to allow the coexistence of two systems under minor intersystem information exchange. The small-cells just need to know what space-frequency block code is used by the macrocell system and no intersystem channels need to be exchanged, contrarily to other schemes recently proposed. Numerical results show that the proposed method achieves a performance close to the case where full cooperation between the tiers is allowed.
\end{abstract}

\section{Introduction}

In order to utilize the limited spectrum efficiently, the concept of heterogeneous networks [1] has been proposed to make the network more agile and flexible by allowing unlicensed users/systems to coexist with the licensed users/systems. In this paper we focus on the heterogeneous network scenario, where the small-cells coexist with macrocells. Small-cells are low powered base stations that are increasingly recognized by the operators [2], as a way to cope with the projected demand for higher data rates for the next generation wireless cellular networks. Other than the capability of providing higher data rates and capacity improvements, small-cells provide advantages, such as the following: they are easy to deploy and have low deployment cost and improved coverage (especially in indoor environment) and are energy efficient [3].

Nevertheless, due to the expected mass deployment of small-cells within the coverage area of a macrocell and the cost involved in acquiring additional spectrum licenses for small-cells, it is likely that the two systems will coexist over the same frequency bands. However, the coexistence of smallcells within the coverage area of a macrocell leads to crosstier/intersystem interference [4]. Also, in a coexistence scenario, the existing macrocell system has the priority to access the radio spectrum and is known as licensed users/systems, whereas the small-cell system can only opportunistically access the free space resources of the macrocell system, without causing interference to it and is known as unlicensed users/systems. In this context, the wireless networks require more careful and dynamic planning and if the two systems are not carefully designed, considerable interference can be generated, thus degrading the performance of both the systems [5].

In order to mitigate interference in the considered heterogeneous networks, different interference management techniques have been proposed. Among them we have resource (time/frequency/space) partitioning [6], power control [7], and pattern nulling/beam steering [8]. In [8] the authors have considered an underlay cognitive radio (CR) uplink scenario, where both primary and secondary systems have two antennas. Secondary users utilize the two antennas in 
order to insert a null in the direction of primary base station (BS), thus protecting the primary users from secondary interference. On the other hand, two antennas are used by primary users in order to perform beam steering in the direction of a primary BS. One recent and effective solution to deal with interference in two-tiered networks is the interference alignment (IA) technique. IA has emerged as an essential approach in order to achieve the degrees of freedom (DoF) in interference channels [9-11]. In [12] the authors proposed a new IA scheme for the heterogeneous networks with multiple antennas where this scheme successively creates transmit beamforming vectors for the small-cell BSs and for the macro BS assuming that they have different number of transmit antennas. Moreover, the concept of IA and CR has been jointly used, in order to mitigate interference in coexistence networks. In [13] the authors propose using IA in conjunction with CR techniques and developing a novel cross-tier IA scheme for two-tiered heterogeneous networks. The work in [14] studied a novel cognitive IA based scheme for the twotier network, where the scheme takes advantage of the OFDM cyclic prefix.

The problem of limited information exchange has been addressed in some publications $[15,16]$. In [15] the authors analyzed the outage probability when different cooperative nonadaptive feedback/feedforward algorithms are considered. In [16], it was shown that only 1-bit of information exchange, between the macrocells and small-cells, is required to achieve full-diversity order at the macro-cell. These works assumed knowledge of the cross-tier channel at the smallcells. In [17] the authors introduced a practical cognitive communication technique for the uplink that uses IA across multiple antennas in order to mitigate the interference from small-cell user terminals (UTs) towards the macro base station. Three different techniques were proposed to align the interference. Namely, they considered coordinated, static, and uncoordinated IA techniques. The first one achieves the best performance with high feedback requirements, where it protects the primary rate as expected, while the other IA techniques require no feedback but at the expense of $30 \%$ reduction of the primary rate [17]. In order to overcome the limitations of coordinated and uncoordinated-static methods, the authors in [18] have investigated a coordinated twobit IA method for the uplink of heterogeneous networks. In [19] we extended the methods of [18] for the downlink of the heterogeneous network.

In coordinated systems, one of the key aspects is the amount of information that needs to be exchanged between the cooperating nodes [20], in order to define the feedback requirements needed by the network to get the benefits from cooperation. On one hand, a system can achieve the maximum diversity order and best performance if full coordination is allowed between the two systems. On the other hand, when no information is exchanged, the diversity is reduced to minimum as demonstrated in [16-18]. The development of practical schemes that can provide close-tooptimal performance with limited information exchange is of paramount importance. Therefore, in [21] we proposed IA based schemes for the downlink of heterogeneous systems under limited intersystem information exchange. Namely, the main contribution was the development of the coordinated $2 n$-bit approach, where some intersystem channels are quantized. In this paper, to further reduce the information exchange between the two systems, we propose a joint IA and SFBC approach. For this new method, the small-cells just need to sense what space-frequency block code is used by the macrocell system and no intersystem channels need to be exchanged, contrarily to the previous proposed approaches. In [21], we present the combination of IA with the Alamouti code [22] and in this paper we present a general diversityoriented joint IA and SFBC formulation that can be applied for any space-frequency block code.

The reminder of the paper is structured as follows: Section 2 introduces the system and signal models for the macrocell and small-cell system. In Section 3, we present in detail the design of the precoders and IA-filter matrices of our proposed method. Section 4 discusses the performance and feedback requirements of all the methods. In Section 5, we present the numerical results and performance comparison of the proposed method with others from literature. Finally, conclusions are provided in Section 6.

Notations. Bold upper case letters denote matrices; bold lower case letters denote vectors. Operations $(\cdot)^{T}$ and $(\cdot)^{*}$ stand for the Hermitian transpose and conjugate of a matrix, respectively. null(A) denotes a matrix whose columns span the null-space of matrix $(\mathbf{A})$ and $\mathbf{A}=\operatorname{diag}\left(\mathbf{A}_{1}, \ldots, \mathbf{A}_{N}\right)$ a diagonal matrix with entries $\mathbf{A}_{1}, \ldots, \mathbf{A}_{N} \cdot \mathbf{A}_{(n)}$ denotes column $n$ of matrix A. $\sigma^{2}$ denotes the noise variance per subcarrier and $\mathbf{I}$ denotes the identity matrix. $\mathbf{A} \otimes \mathbf{B}$ denotes the Kronecker product between matrices $\mathbf{A}$ and $\mathbf{B} . \mathbf{x}=$ $\operatorname{vec}(\mathbf{X})$ denotes the vectorization of matrix $\mathbf{X}$. For a complex number $a, \mathfrak{R}\{a\}$ and $\mathfrak{\Im}\{a\}$ denote its real and imaginary parts, respectively.

\section{System Model}

Let us consider the downlink of a heterogeneous network, where a set of $K$ small-cells are overlaid within the boundaries of a macrocell, both sharing the same spectrum as depicted in Figure 1. $K$ small-cell access points (SAPs) are able to cooperate through a backhaul network [23] (e.g., radio over fiber) to a central unit (CU) that allows joint processing of transmitted signals. In this work we consider the downlink case, that is, the base station (BS), and access points (APs) are sending information to the corresponding UTs. Furthermore, OFDM based terminals with $N_{c}$ available subcarriers are considered, where transmit power per subcarrier for macro base station (MBS) and SAPs is constraint to $P_{m}$ and $P_{s}$, respectively. We consider that the MBS serves only one user terminal, macro UT (MUT), per subcarrier (considering OFDM/A based system, the total number of macrocell users can be significantly larger than one, since different set of resources can be allocated to different users), and the SAP $k$ serves only the small-cell user terminal $k\left(\mathrm{SUT}_{k}\right) k=$ $\{1, \ldots, K\}$. 


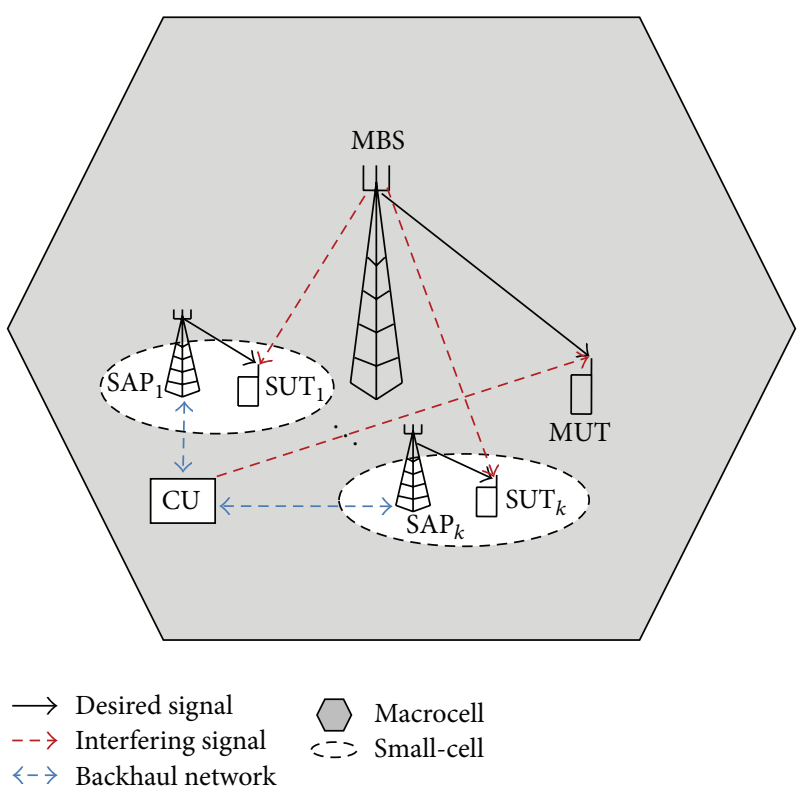

FIGURE 1: System model: small-cells within coverage area of macrocell.

2.1. Macrocell System. For the macrocell system we assume that the MBS and MUT have $M_{m}$ and $N_{m}$ antennas, respectively, as presented in the block diagram of the considered system in Figure 2 . With $f_{n}$ indicating the subcarrier index, if $\mathbf{x}_{m}^{f_{n}}$ is the transmit vector then the received signal $\mathbf{y}_{m}^{f_{n}}$ is given by

$$
\mathbf{y}_{m}^{f_{n}}=\mathbf{G}_{1}^{f_{n}} \mathbf{x}_{m}^{f_{n}}+\mathbf{G}_{2}^{f_{n}} \mathbf{x}_{s}^{f_{n}}+\mathbf{n}_{m}^{f_{n}}
$$

where $\mathbf{x}_{s}^{f_{n}} \in \mathbb{C}^{M_{s} K}, \mathbf{G}_{1}^{f_{n}} \in \mathbb{C}^{N_{m} \times M_{m}}, \mathbf{G}_{2}^{f_{n}} \in \mathbb{C}^{N_{m} \times M_{s} K}$, and $\mathbf{n}_{m}^{f_{n}} \in \mathbb{C}^{N_{m}}$ denote the overall transmitted signal at the smallcells, the channel between MBS and MUT, the overall channel between CU and MUT (i.e., the channels between the SAPs and the MUT), and the zero-mean white Gaussian noise with variance $\sigma^{2}$, respectively. We consider that at the macrocell BS only $\mathbf{G}_{1}^{f_{n}}$ is known and it has no information about the small-cell system. Furthermore, we assume that the MUT is a mobile terminal and then $\mathbf{G}_{1}^{f_{n}}$ changes on every Transmission Time Interval (TTI).

We consider a block fading MIMO channel; that is $\mathbf{G}_{1}^{f_{n}}=$ $\mathbf{G}_{1}$ for $f_{n}=1, \ldots, F$, and the channel is independent between different blocks of $F$ subcarriers. Thus, the system equation over one block is [24]

$$
\mathbf{Y}_{m}=\mathbf{G}_{1} \mathbf{X}_{m}+\mathbf{I}_{s}+\mathbf{N}_{m}
$$

where $\mathbf{Y}_{m}=\left[\mathbf{y}_{m}^{1}, \ldots, \mathbf{y}_{m}^{F}\right]$ is the received signal matrix, $\mathbf{X}_{m}=$ $\left[\mathbf{x}_{m}^{1}, \ldots, \mathbf{x}_{m}^{F}\right]$ is the transmitted signal, $\mathbf{I}_{s}=\left[\mathbf{G}_{2}^{1} \mathbf{x}_{s}^{1}, \ldots, \mathbf{G}_{2}^{F} \mathbf{x}_{s}^{F}\right]$ is the intertier interference, and $\mathbf{N}_{m}=\left[\mathbf{n}_{m}^{1}, \ldots, \mathbf{n}_{m}^{F}\right]$ is the zero-mean white Gaussian noise with variance $\sigma^{2}$.
The macrocell system employs SFBC to encode $S_{m}$ complex symbols $d_{m}^{1}, \ldots, d_{m}^{S_{m}}$ chosen from $r$-QAM constellation. We consider linear dispersion (LD) codes of the form [25]

$$
\mathbf{X}_{m}=\sum_{s=1}^{S_{m}}\left(\mathbf{A}_{m}^{s} \mathfrak{R}\left\{d_{m}^{s}\right\}+\mathbf{B}_{m}^{s} \mathfrak{\Im}\left\{d_{m}^{s}\right\}\right),
$$

where $d_{m}^{s}=\mathfrak{R}\left\{d_{m}^{s}\right\}+j \mathfrak{\Im}\left\{d_{m}^{s}\right\}, m=1, \ldots, S_{m}$, and $\mathbf{A}_{m}^{s}$ and $\mathbf{B}_{m}^{s}$ are the codeword matrices. The rate of the LD code is

$$
R=\frac{S_{m}}{F} \log _{2}(r) \text { (bits/subcarrier). }
$$

Therefore, by rewriting (2) in column-stacked form we obtain

$$
\mathbf{y}_{m}=\left(\mathbf{I}_{F} \otimes \mathbf{G}_{1}\right) \mathbf{x}_{m}+\mathbf{i}_{s}+\mathbf{n}_{m}=\mathscr{G}_{1} \mathbf{V}_{m} \mathbf{d}_{m}+\mathbf{i}_{s}+\mathbf{n}_{m},
$$

where $\mathscr{G}_{1}=\mathbf{I}_{F} \otimes \mathbf{G}_{1}, \mathbf{x}=\operatorname{vec}(\mathbf{X})$ is $N_{m} F$ dimensional, $\mathbf{i}_{s}=\operatorname{vec}\left(\mathbf{I}_{s}\right)$ is $M_{m} F$ dimensional, $\mathbf{x}_{m}=\operatorname{vec}\left(\mathbf{X}_{m}\right)=\mathbf{V}_{m} \mathbf{d}_{m}$ is $M_{m} F$ dimensional, $\mathbf{d}_{m}=\left[\mathfrak{R}\left\{d_{m}^{1}\right\}, \ldots, \mathfrak{R}\left\{d_{m}^{S_{m}}\right\}, \mathfrak{\Im}\left\{d_{m}^{1}\right\}, \ldots\right.$, $\left.\mathfrak{\Im}\left\{d_{m}^{S_{m}}\right\}\right]^{T}$, and $\mathbf{V}_{m}=\left[\operatorname{vec}\left(\mathbf{A}_{1}\right), \ldots, \operatorname{vec}\left(\mathbf{A}_{S_{m}}\right), \operatorname{vec}\left(\mathbf{B}_{1}\right), \ldots\right.$, $\operatorname{vec}\left(\mathbf{B}_{S_{m}}\right)$ ] is $N_{m} F \times 2 S_{m}$ code generator matrix that is an equivalent representation of the LD code.

2.2. Small-Cell System. At the small-cell system, each SAP has $M_{s}$ transmit and the $\mathrm{SUT}_{k} k=\{1, \ldots, K\}$ has $N_{s}$ receive antennas as shown in Figure 2. If $\mathbf{x}_{s}^{f_{n}}$ is the transmitted signal vector, at the CU on subcarrier $f_{n}$ the received signal at $\mathrm{SUT}_{k}$ is given by

$$
\mathbf{y}_{s k}^{f_{n}}=\mathbf{F}_{k}^{f_{n}} \mathbf{x}_{m}^{f_{n}}+\mathbf{H}_{k}^{f_{n}} \mathbf{x}_{s}^{f_{n}}+\mathbf{n}_{s k}^{f_{n}}
$$

where $\mathbf{F}_{k}^{f_{n}} \in \mathbb{C}^{N_{s} \times M_{m}}, \mathbf{H}_{k}^{f_{n}} \in \mathbb{C}^{N_{s} \times M_{s} K}$, and $\mathbf{n}_{s k}^{f_{n}} \in \mathbb{C}^{N_{s}}$ denote the channel between the MBS and $\mathrm{SUT}_{k}$, the overall channel between the SAPs and $\mathrm{SUT}_{k}$, and the zero-mean white Gaussian noise with variance $\sigma^{2}$ at $\mathrm{SUT}_{k}$, respectively. In the following, we assume that the SUTs are low mobility terminals (since the terminals associated with the small-cells are mainly indoor/pedestrian users) and then channel $\mathbf{F}_{k}^{f_{n}}$ can be considered as quasi-static which reduces the overhead required for its estimation.

Using a similar procedure as in the previous section we obtain [24]

$$
\mathbf{y}_{s k}=\mathscr{F}_{k} \mathbf{V}_{m} \mathbf{d}_{m}+\mathscr{H}_{k} \mathbf{x}_{s}+\mathbf{n}_{m}
$$

where $\mathbf{y}_{s k}=\left[\left(\mathbf{y}_{s k}^{1}\right)^{T}, \ldots,\left(\mathbf{y}_{s k}^{F}\right)^{T}\right]^{T}, \mathscr{F}_{k}=\operatorname{diag}\left(\mathbf{F}_{k}^{1}, \ldots, \mathbf{F}_{k}^{F}\right)$, $\mathscr{H}_{k}=\operatorname{diag}\left(\mathbf{H}_{k}^{1}, \ldots, \mathbf{H}_{k}^{F}\right), \mathbf{x}_{s}=\left[\left(\mathbf{x}_{s}^{1}\right)^{T}, \ldots,\left(\mathbf{x}_{s}^{F}\right)^{T}\right]^{T}$, and $\mathbf{n}_{s k}=$ $\left[\left(\mathbf{n}_{s k}^{1}\right)^{T}, \ldots,\left(\mathbf{n}_{s k}^{F}\right)^{T}\right]^{T}$.

To compute the CU transmit signal a linear precoder is considered; that is, the CU transmits

$$
\mathbf{x}_{s}=\mathbf{V}_{s} \mathbf{d}_{s}
$$

where $\mathbf{V}_{s} \in \mathbb{C}^{M_{s} K F \times S_{s} K F}, \mathbf{d}_{s}=\left[\mathbf{d}_{s k}^{f_{n}}\right]_{1 \leq k \leq K, 1 \leq f_{n} \leq F} \in \mathbb{C}^{S_{s} K F}$, and $\mathbf{d}_{s k}^{f_{n}} \in \mathbb{C}^{S_{s}}$ denote the overall precoder computed at the CU, the concatenation of $K$ SAPs transmit symbols, and $\mathbf{d}_{s k}^{f_{n}}$ SAP 


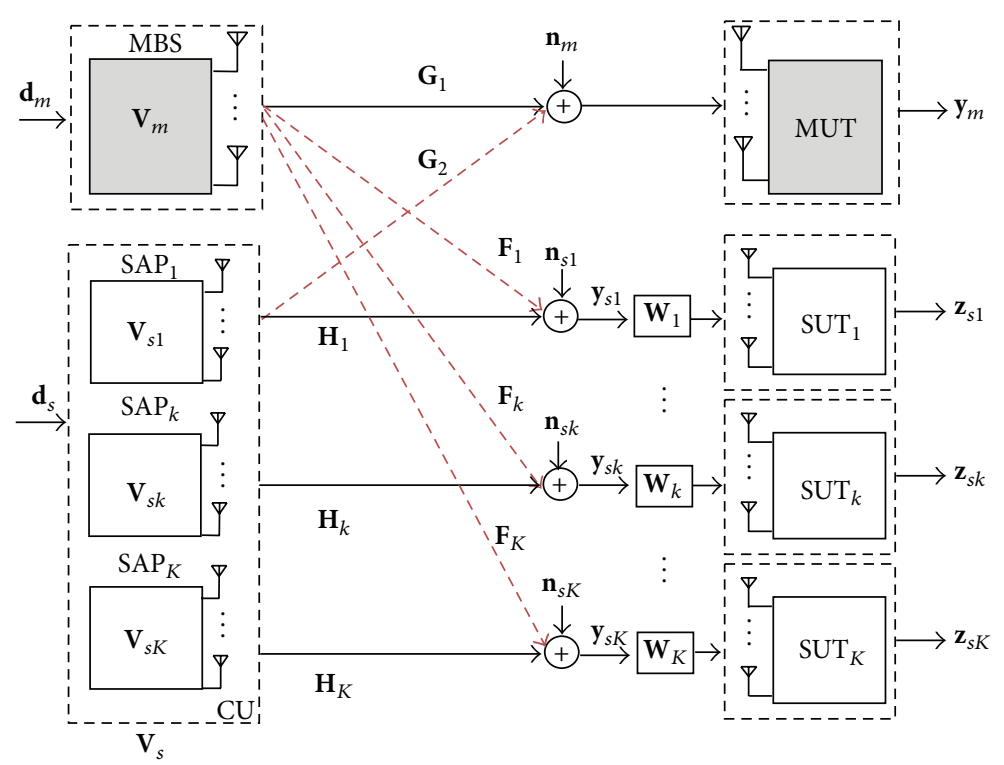

FIGURE 2: Block diagram of the considered system.

$k$ transmit symbols, respectively. The transmit power at the $\mathrm{CU}$ is constrained to $P_{s}$, per subcarrier

$$
\operatorname{tr}\left(\mathbf{V}_{s}^{f_{n}^{H}} \mathbf{V}_{s}^{f_{n}}\right) \leq P_{s}
$$

and the received signal after filter matrix $\left(\mathbf{W}_{k}\right)$ at the $\mathrm{SUT}_{k}$, by taking into account (7) and (8), is

$$
\mathbf{z}_{s k}=\mathbf{W}_{k}\left(\mathscr{F}_{k} \mathbf{V}_{m} \mathbf{d}_{m}+\mathscr{H}_{k} \mathbf{V}_{s} \mathbf{d}_{s}+\mathbf{n}_{m}\right) \text {. }
$$

\section{Proposed Method: IA-Filter Matrices Design}

In the following, we present the design of our proposed joint IA and SFBC scheme with the aim of completely eliminating the need for information exchange between the macrocell and small-cell systems. As seen in the full-coordinated and coordinated 2n-bit methods [21], the design of the precoder at the MBS and IA-filter matrix at the SUTs depends on channel $\mathbf{G}_{1}$ between the MBS and MUT. The key idea behind the use of SFBC schemes at the macrocell system is that it allows the design of the IA-filter matrix at the SUTs without having any coordination between the two systems. More specifically, the small-cells just need to sense that the macrocell system is using a scheme based on a SFBC.

3.1. IA-Filter Matrix Design at SUT. In this section, we present the design of IA-filter matrix at the SUTs for the proposed joint IA and SFBC scheme. We consider that the macrocell system has no information about the existence of small-cells within its coverage area. In the coexistence scenario, the MBS interferes with the SUTs. From (10) we can see that to enforce the zero-interference condition and mitigate the interference coming from MBS the IA-filter matrix at $\mathrm{SUT}_{k}$ must satisfy

$$
\mathbf{W}_{k} \mathscr{F}_{k} \mathbf{V}_{m}=\mathbf{0} \text {. }
$$

In order to cancel the interference coming from MBS towards the $\mathrm{SUT}_{k}$, we need to compute an appropriate filter matrix at the $\mathrm{SUT}_{k}$. From (11) it follows that to satisfy the zerointerference condition the IA-filter matrix at $\mathrm{SUT}_{k}$ is

$$
\mathbf{W}_{k}=\operatorname{null}\left(\mathscr{F}_{k} \mathbf{V}_{m}\right) \text {. }
$$

As seen in Section 2.1, precoder $\mathbf{V}_{m}$ for SFBCs does not depend on the macrochannel, and thus there is no need to exchange any information from the macrocell to the smallcell system to design the IA-filter matrix, contrarily to the full-coordinated and coordinated 2n-bit methods [21]. For these two cases the precoder is computed for each channel instance and as the macrocell terminal is a mobile terminal equalizer matrix $\mathbf{W}_{k}$ must be computed on every TTI. This means that the IA-filter matrix must be exchanged between the two systems every TTI. Another possible strategy consists of estimating the equivalent channel $\mathbf{F}_{k}^{f_{n}} \mathbf{V}_{m}^{f_{n}}$, by listening to the pilot signals, but it will also require a high pilot density [26].

After applying the IA-filter matrix mentioned in (12) to (10), we obtain

$$
\begin{aligned}
\mathbf{z}_{s k} & =\mathbf{W}_{k}\left(\mathscr{F}_{k} \mathbf{V}_{m} \mathbf{d}_{m}+\mathscr{H}_{k} \mathbf{V}_{s} \mathbf{d}_{s}+\mathbf{n}_{m}\right) \\
& =\mathbf{W}_{k} \mathscr{F}_{k} \mathbf{V}_{m} \mathbf{d}_{m}+\mathbf{W}_{k} \mathbf{n}_{m} .
\end{aligned}
$$

From (11) and (13) we verify that the interference from MBS is completely removed at SUTs. This is made possible due to the redundancy present in the MBS transmitted data symbols.

3.2. Small-Cells-to-Macrocell Interference Cancelation. In the previous section we considered the interference from the macrocells to the small-cells. Now we describe how to handle the interference from the small-cells to the macrocell. The small-cell system should not interfere with the macro-cell system (i.e., the macrocell has priority to access the available resources). Also the SUTs should not interfere with each other. We consider that the SAPs are connected via the 


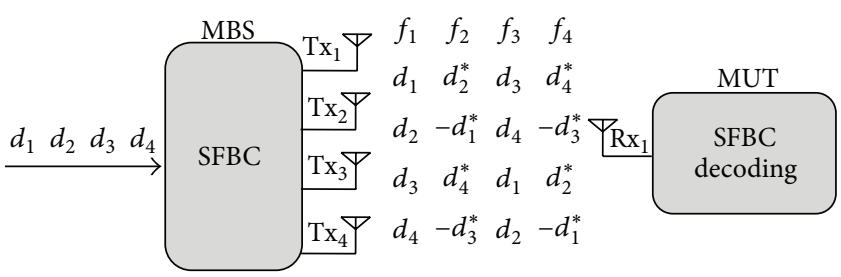

FIgURE 3: Quasi-orthogonal codes at MBS.

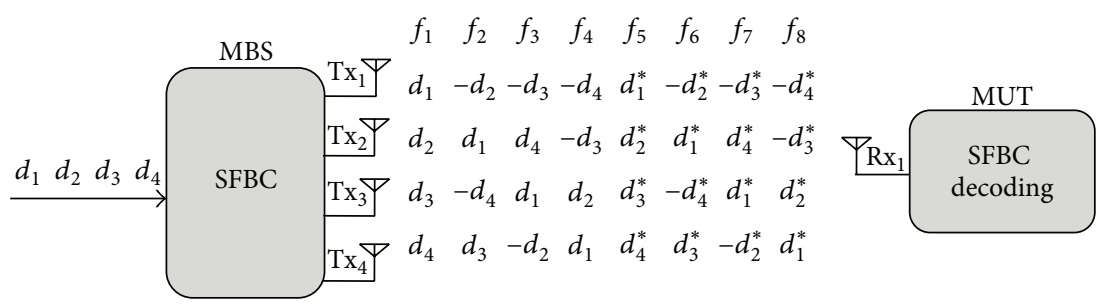

Figure 4: Tarokh codes at MBS.

backhaul network to CU in order to perform joint processing of transmitted signals. The CU has enough DoF (i.e., $K M_{s}$ ) to cancel both the interference that the SAPs cause in the MUT and the interference between SUTs. The precoding matrix at the $\mathrm{CU}$ is based on the $\mathrm{ZF}$ criteria. In this context, $\mathrm{ZF}$ precoder $\mathbf{V}_{s}^{f_{n}}$, computed at the $\mathrm{CU}$, is given by

$$
\mathbf{V}_{s}^{f_{n}}=\mathbf{A}^{f_{n}^{H}}\left(\mathbf{A}^{f_{n}} \mathbf{A}^{f_{n}^{H}}\right)^{-1}, \quad f_{n}=1, \ldots, F,
$$

where $\mathbf{A}^{f_{n}}=\mathbf{W}^{f_{n}} \mathbf{H}_{\text {eq }}^{f_{n}}, \mathbf{H}_{\text {eq }}^{f_{n}}=\left[\left(\mathbf{G}_{2}^{f_{n}}\right)^{H},\left(\mathbf{H}_{1}^{f_{n}}\right)^{H}, \ldots,\left(\mathbf{H}_{K}^{f_{n}}\right)^{H}\right]^{H}$, and $\mathbf{W}^{f_{n}}=\operatorname{diag}\left(\mathbf{I}, \mathbf{W}_{1}^{f_{n}}, \ldots, \mathbf{W}_{k}^{f_{n}}, \ldots, \mathbf{W}_{K}^{f_{n}}\right)$. Filter matrix $\mathbf{W}_{k}^{f_{n}}$ is known at the CU since channels $\mathbf{F}_{k}^{f_{n}}$ are quasi-static; the SUTs may feed back them to the CU without much overhead requirements.

3.3. Examples for Specific SFBC Codes. In the following, we just provide few examples of diversity-oriented SFBC schemes used at MBS in order to design the IA-filter matrix of our proposed scheme. We considered two SFBC schemes: quasi-orthogonal codes [27] and Tarokh codes [28] with the data symbols coded in space and frequency as shown in Figures 3 and 4, respectively. Furthermore, from the context of space-time/space-frequency coding literature, the channel between adjacent carriers is assumed to be approximately constant (OFDM based systems are usually designed so that channels between some adjacent carriers are approximately flat); that is, $\mathbf{G}_{1}^{f_{m}} \approx \mathbf{G}_{1}^{f_{n}}, m \neq n \in \mathbb{N}$.

3.3.1. Quasi-Orthogonal Codes. As seen in [21], the Alamouti scheme is restricted to 2 antennas at the transmitter side. Therefore, we consider the quasi-orthogonal based scheme to be able to use more than 2 transmit antennas and increase the multiplexing gain. We assume that the transmitter has four antennas $\left(M_{m}=4\right)$ and the receiver has a single antenna $\left(N_{m}=1\right)$, as shown in Figure 3. With this method four pairs of four data symbols are transmitted in parallel. The four data symbols are transmitted over four antennas on four subcarriers, $F=4$, according to the following encoding:

$$
\begin{aligned}
& \mathbf{x}_{m}^{f_{1}}= {\left[\begin{array}{l}
d_{1} \\
d_{2} \\
d_{3} \\
d_{4}
\end{array}\right], } \\
& \mathbf{x}_{m}^{\left(f_{2}\right)^{*}}=\left[\begin{array}{c}
d_{2} \\
-d_{1} \\
d_{4} \\
-d_{3}
\end{array}\right], \\
& \mathbf{x}_{m}^{f_{3}}=\left[\begin{array}{c}
d_{3} \\
d_{4} \\
d_{1} \\
d_{2}
\end{array}\right], \\
& \mathbf{x}_{m}^{\left(f_{4}\right)^{*}}=\left[\begin{array}{c}
d_{4} \\
-d_{3} \\
d_{2} \\
-d_{1}
\end{array}\right] .
\end{aligned}
$$

For this case, as mentioned previously, the MBS precoder is applied jointly for $F=4$ consecutive subcarriers:

$$
\begin{aligned}
& \mathbf{V}_{m}^{T} \\
& =\left[\begin{array}{cccccccccccccccc}
1 & 0 & 0 & 0 & 0 & -1 & 0 & 0 & 0 & 0 & 1 & 0 & 0 & 0 & 0 & -1 \\
0 & 1 & 0 & 0 & 1 & 0 & 0 & 0 & 0 & 0 & 0 & 1 & 0 & 0 & 1 & 0 \\
0 & 0 & 1 & 0 & 0 & 0 & 0 & -1 & 1 & 0 & 0 & 0 & 0 & -1 & 0 & 0 \\
0 & 0 & 0 & 1 & 0 & 0 & 1 & 0 & 0 & 1 & 0 & 0 & 1 & 0 & 0 & 0 \\
j & 0 & 0 & 0 & 0 & j & 0 & 0 & 0 & 0 & j & 0 & 0 & 0 & 0 & j \\
0 & j & 0 & 0 & -j & 0 & 0 & 0 & 0 & 0 & 0 & j & 0 & 0 & -j & 0 \\
0 & 0 & j & 0 & 0 & 0 & 0 & j & j & 0 & 0 & 0 & 0 & j & 0 & 0 \\
0 & 0 & 0 & j & 0 & 0 & -j & 0 & 0 & j & 0 & 0 & -j & 0 & 0 & 0
\end{array}\right] .
\end{aligned}
$$


As can be verified from (16) the macrocell precoder does not depend on the macrochannel and this means there is no need to exchange any channel information from the macrocell to the small-cell system to design the IA-filter matrix.

3.3.2. Tarokh Codes. Once again, for this case, we assume that the transmitter has four antennas $\left(M_{m}=4\right)$ and the receiver has a single antenna $\left(N_{m}=1\right)$, as shown in Figure 4 . The only difference is the number of subcarriers used to transmit the data symbols; for this case eight subcarriers are used, that is, the Tarokh code that provides the code rate of $1 / 2$. The four data symbols are transmitted over four antennas on eight subcarriers, $F=8$, according to the following encoding:

$$
\begin{gathered}
\mathbf{x}_{m}^{f_{1}}=\left[\begin{array}{l}
d_{1} \\
d_{2} \\
d_{3} \\
d_{4}
\end{array}\right], \\
\mathbf{x}_{m}^{f_{2}}=\left[\begin{array}{c}
-d_{2} \\
d_{1} \\
-d_{4} \\
d_{3}
\end{array}\right], \\
\mathbf{x}_{m}^{f_{3}}=\left[\begin{array}{c}
-d_{3} \\
d_{4} \\
d_{1} \\
-d_{2}
\end{array}\right],
\end{gathered}
$$

$$
\mathbf{x}_{m}^{f_{4}}=\left[\begin{array}{c}
-d_{4} \\
-d_{3} \\
d_{2} \\
d_{1}
\end{array}\right]
$$

$$
\mathbf{x}_{m}^{\left(f_{5}\right)^{*}}=\left[\begin{array}{l}
d_{1} \\
d_{2} \\
d_{3} \\
d_{4}
\end{array}\right],
$$

$$
\mathbf{x}_{m}^{\left(f_{6}\right)^{*}}=\left[\begin{array}{c}
-d_{2} \\
d_{1} \\
-d_{4} \\
d_{3}
\end{array}\right] \text {, }
$$

$$
\mathbf{x}_{m}^{\left(f_{7}\right)^{*}}=\left[\begin{array}{c}
-d_{3} \\
d_{4} \\
d_{1} \\
-d_{2}
\end{array}\right],
$$

$$
\mathbf{x}_{m}^{\left(f_{8}\right)^{*}}=\left[\begin{array}{c}
-d_{4} \\
-d_{3} \\
d_{2} \\
d_{1}
\end{array}\right] \text {. }
$$

For the Tarokh codes, the MBS precoder is applied jointly for $F=8$ consecutive subcarriers as

$$
\begin{aligned}
& \mathbf{V}_{m}^{T} \\
& =\left[\begin{array}{cccccccccccccccccccccccccccccccccc}
1 & 0 & 0 & 0 & 0 & 1 & 0 & 0 & 0 & 0 & 1 & 0 & 0 & 0 & 0 & 1 & 1 & 0 & 0 & 0 & 0 & 1 & 0 & 0 & 0 & 0 & 1 & 0 & 0 & 0 & 0 & 1 \\
0 & 1 & 0 & 0 & -1 & 0 & 0 & 0 & 0 & 0 & 0 & -1 & 0 & 0 & 1 & 0 & 0 & 1 & 0 & 0 & -1 & 0 & 0 & 0 & 0 & 0 & 0 & -1 & 0 & 0 & 1 & 0 \\
0 & 0 & 1 & 0 & 0 & 0 & 0 & 1 & -1 & 0 & 0 & 0 & 0 & -1 & 0 & 0 & 0 & 0 & 1 & 0 & 0 & 0 & 0 & 1 & -1 & 0 & 0 & 0 & 0 & -1 & 0 & 0 \\
0 & 0 & 0 & 1 & 0 & 0 & -1 & 0 & 0 & 1 & 0 & 0 & -1 & 0 & 0 & 0 & 0 & 0 & 0 & 1 & 0 & 0 & -1 & 0 & 0 & 1 & 0 & 0 & -1 & 0 & 0 & 0 \\
j & 0 & 0 & 0 & 0 & j & 0 & 0 & 0 & 0 & j & 0 & 0 & 0 & 0 & j & -j & 0 & 0 & 0 & 0 & -j & 0 & 0 & 0 & 0 & -j & 0 & 0 & 0 & 0 & -j \\
0 & j & 0 & 0 & -j & 0 & 0 & 0 & 0 & 0 & 0 & -j & 0 & 0 & -j & 0 & 0 & -j & 0 & 0 & j & 0 & 0 & 0 & 0 & 0 & 0 & j & 0 & 0 & -j & 0 \\
0 & 0 & j & 0 & 0 & 0 & 0 & j & -j & 0 & 0 & 0 & 0 & -j & 0 & 0 & 0 & 0 & -j & 0 & 0 & 0 & 0 & -j & j & 0 & 0 & 0 & 0 & j & 0 & 0 \\
0 & 0 & 0 & j & 0 & 0 & -j & 0 & 0 & j & 0 & 0 & -j & 0 & 0 & 0 & 0 & 0 & 0 & -j & 0 & 0 & j & 0 & 0 & -j & 0 & 0 & j & 0 & 0 & 0
\end{array}\right] .
\end{aligned}
$$

As in the quasi-orthogonal codes, the precoder is also constant and not dependent on the macrocell channel as verified in (18), where this condition enables the design of IAfilter at SUTs without any information exchange between the two systems.

\section{Performance and Information Exchange Requirement Comparison}

As it will be verified from the numerical results section, the full-coordinated method [19] achieves the best performance but it has the highest intersystem information sharing 
TABLE 1: Comparison of intersystem information exchange and performance.

\begin{tabular}{lcc}
\hline Methods & Information exchange requirements & Performance \\
\hline Full-coordinated & $2 M_{m} N_{m} N_{c}$ real number & Optimal performance \\
Uncoordinated-static & 0 & Worst performance \\
Coordinated $2 n$-bit & $2 n M_{m} N_{m} N_{c}$ bits & Close to optimal \\
Joint IA and SFBC scheme & 0 & Much better than uncoordinated-static method \\
\hline
\end{tabular}

requirements, since the macrocell system must coordinate with the small-cell system every TTI, in order to share $2 M_{m} N_{m}$ real numbers, per subcarrier. For OFDM based system $2 M_{m} N_{m} N_{c}$ reals can be a huge number that substantially increases the feedback requirements. The uncoordinatedstatic method [19] does not require any information exchange between the two systems, but this results in significant performance degradation for the macrocell system. To achieve a good trade-off between performance and information exchange requirements, we recently proposed a coordinated $2 n$-bit method [21] that enables a significant reduction in the information exchange requirements, that is, from $2 M_{m} N_{m} N_{c}$ real numbers to $2 n M_{m} N_{m} N_{c}$ bits, and achieves close-to-fullcoordinated performance.

However, pursuing for methods that can achieve performance close to the full-coordinated method without almost any information exchange is of great importance. Therefore, we propose joint IA with SFBC scheme where no channel information needs to be exchanged between the two systems; that is, these approaches have the same requirements as the uncoordinated-static approach, but with a performance close to the full-coordinated method. A summary of the intersystem information exchange requirements is presented in Table 1.

\section{Numerical Results and Performance Comparison}

This section provides the performance assessment of our proposed method. We compare it to the full-coordinated, uncoordinated-static, coordinated $2 n$-bit (in this paper we consider $n=1$ ), and joint IA and Alamouti code methods with the help of numerical simulations. We consider the scenario with 2 small-cells (i.e., $K=2$ ) within the coverage area of MBS but adding more small-cells will not impact on the performance of the macrocell system, since the interference can be completely eliminated irrespective of the number of small-cells. We assume that the SAPs are able to cooperate through a backhaul network to CU to perform joint processing of signals. We assume that the number of antennas at the MBS is 4 and each SAP and each SUT has 2 antennas and single antenna at the MUT; that is $M_{m}=4, M_{s}=N_{s}=$ 2 , and $N_{m}=1$.

We consider the ITU pedestrian channel model B, with modified tap delays according to the sampling frequency specified in LTE standards. The SNR at the cell edge is defined as $\left(P_{t} / \sigma^{2}\right)$, where $P_{t}$ is the transmit power, and it is equal to $P_{m}=1$, if the Bit-Error Rate (BER) is measured for the macrocell system, or it can be equal to $P_{s}=1$, if the BER is

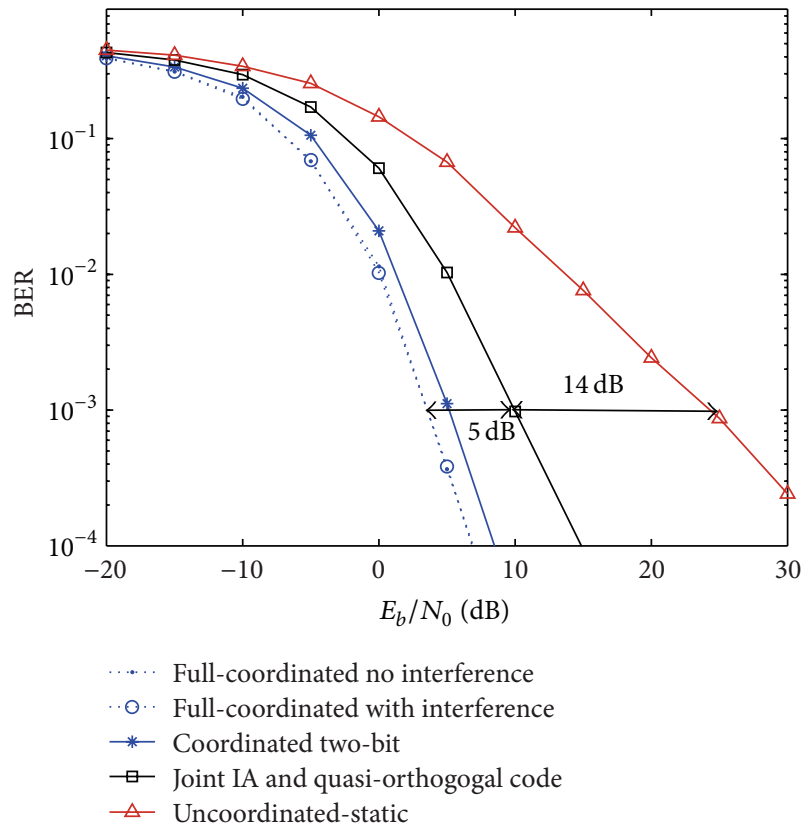

FIGURE 5: BER performance for the macrocell system.

calculated for the small-cell system. The OFDM parameters used for simulating both the macrocell and small-cell systems are FFT size $=1024$ (where only 128 subcarriers are used for both the systems); sampling frequency $f_{s}=15.36 \mathrm{MHz}$; cyclic prefix length $c_{p}=5.21 \mu \mathrm{s}$; and subcarrier separation is $15 \mathrm{kHz}$.

We present results for two joint IA and SFBCs: IA with a quasi-orthogonal code [27] and IA with a half-rate orthogonal Tarokh code [28]. In order to allow an appropriate comparison, all the considered methods are evaluated for the same spectral efficiency. Therefore, we used QPSK modulation for joint IA and quasi-orthogonal code, coordinated twobit, full-coordinated, and uncoordinated-static schemes, as well as 16-QAM for the joint IA and Tarokh codes.

Let us start by considering the case where IA is combined with the quasi-orthogonal code. For this case we compare the performance of the full-coordinated (for both the case of macrocell/small-cell coexistence and the case where small-cell system is switched off), coordinated twobit, uncoordinated-static, and joint IA and quasi-orthogonal code methods. In Figures 5 and 6, we present the BER performance for the macrocell and small-cell system, respectively (using QPSK modulation for all the curves). From Figure 5, we can notice that the coordinated two-bit approach provides close-to-optimal performance (a gap of around $2 \mathrm{~dB}$ 


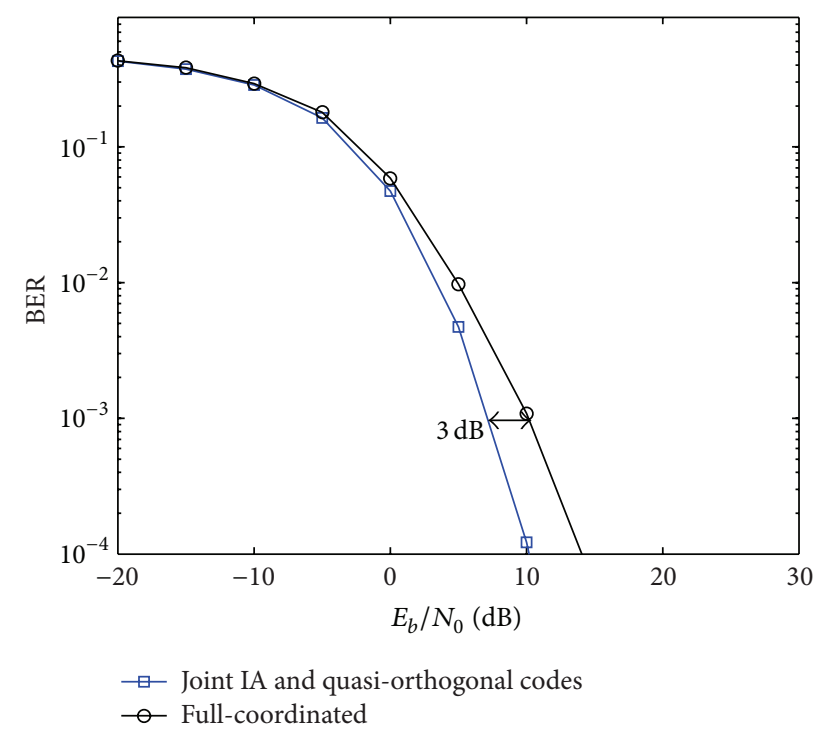

FIGURE 6: BER performance for the small-cell system.

for a target BER of $10^{-3}$ ). On the other hand, the performance of joint IA and quasi-orthogonal code approach has a gap of around $5 \mathrm{~dB}$ as compared to the full-coordinated method and achieves much better performance (a gap of around $14 \mathrm{~dB}$ for a target BER of $10^{-3}$ ) as compared to the uncoordinatedstatic scheme, even if the information exchange requirements of both the schemes are identical.

In Figure 6, we compare the BER performance of the proposed joint IA and quasi-orthogonal code with the fullcoordinated method for the small-cell system. The proposed joint IA and quasi-orthogonal code scheme provides around $3 \mathrm{~dB}$ better performance as compared to the case where full coordination is allowed between the two tiers.

Let us now consider the case where IA is combined with the half-rate orthogonal Tarokh code. We compare the performance of the full-coordinated, coordinated two-bit, uncoordinated-static, and joint IA and Tarokh code methods. In Figures 7 and 8, we present the BER performance for the macrocell and small-cell system, respectively (using QPSK modulation for full-coordinated and coordinated two-bit and uncoordinated-static curves and 16-QAM modulation for the joint IA and Tarokh code curve). From the curves presented in Figure 7, we can see that yet again the coordinated two-bit approach provides quite close-to-optimal performance. On the other hand, the performance of joint IA and Tarokh code approach has a gap of around $3 \mathrm{~dB}$ as compared to the fullcoordinated method and achieves much better performance (a gap of around $18 \mathrm{~dB}$ for a target BER of $10^{-3}$ ) as compared to the uncoordinated-static scheme, even if the information exchange requirements of both the schemes are identical as seen in the previous case.

In Figure 8, we compare the BER performance of the proposed joint IA and Tarokh code with the full-coordinated method for the small-cell system. The performance of proposed joint IA and Tarokh code scheme is around $1 \mathrm{~dB}$ better as compared to the full-coordinated case.

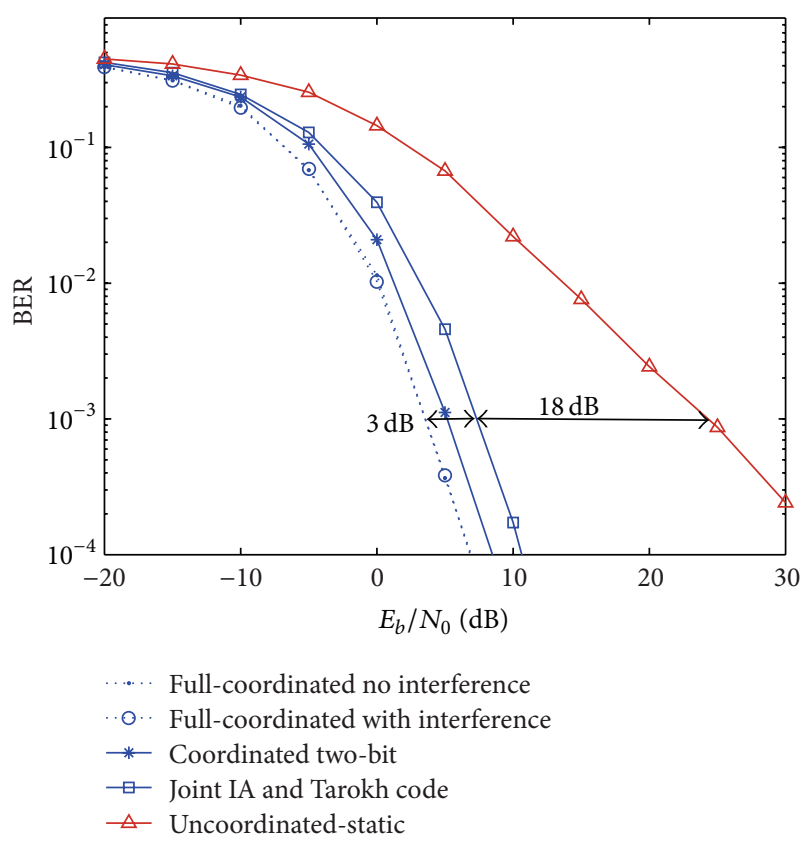

FIGURE 7: BER performance for the macrocell system.

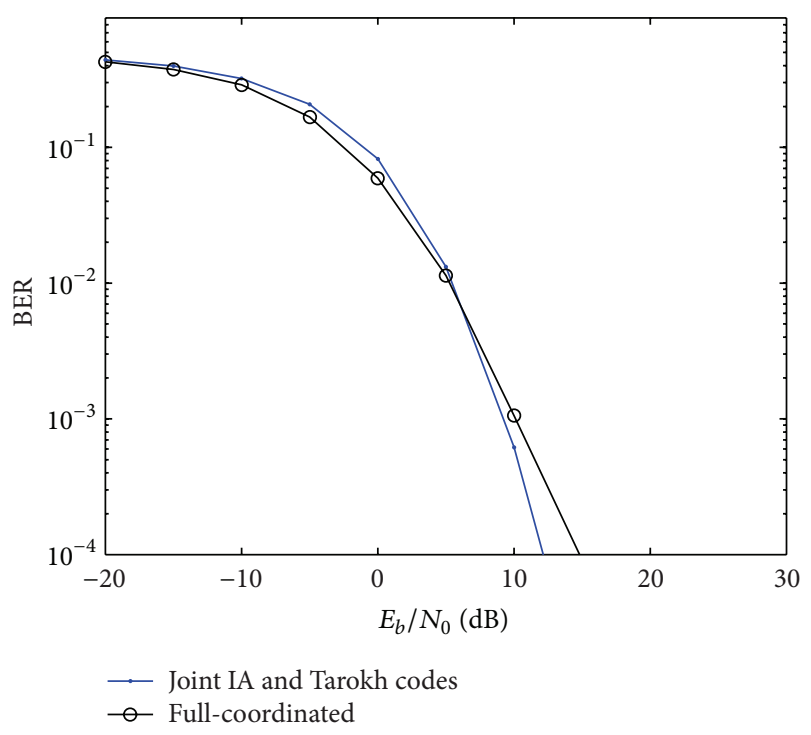

FIGURE 8: BER performance for the small-cell system.

In Figures 9 and 10, we compare the performance of SFBC schemes at the macrocell and small-cell systems, respectively. We also added the curve for IA with the Alamouti code (the simplest SFBC). As it can be verified from Figure 9, the joint IA and Tarokh code provides the best performance as compared to the joint IA and Alamouti code/quasi-orthogonal code (i.e., a gap of around $3 \mathrm{~dB}$ and $6 \mathrm{~dB}$, resp.). At the small-cell system, the performance of joint IA and Alamouti code/joint IA and quasi-orthogonal code is identical and the performance of joint IA and Tarokh code is around $2 \mathrm{~dB}$ worse as compared to the other two schemes as shown in Figure 10. This is due to the fact that the high order 


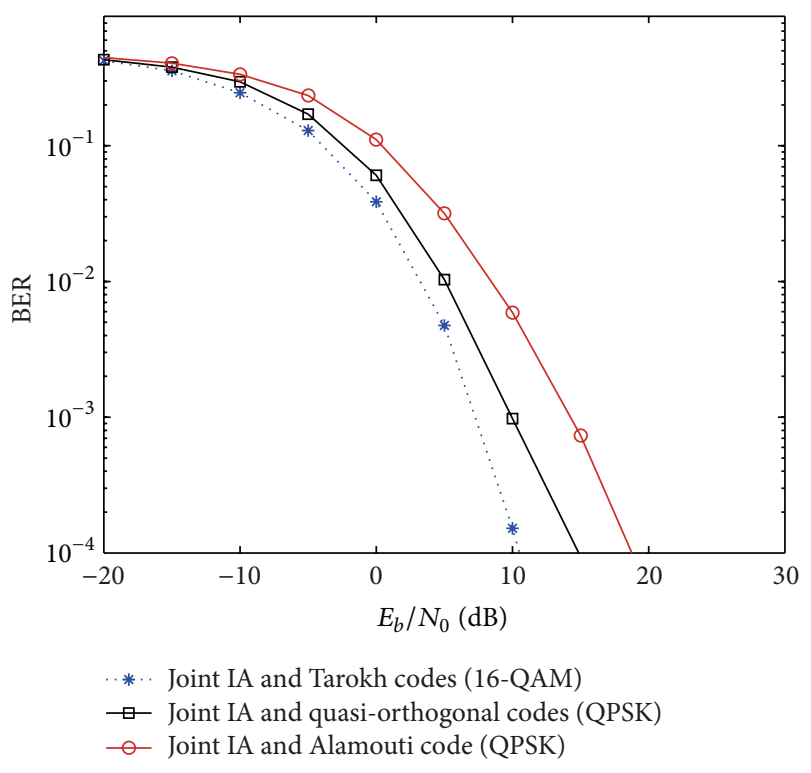

FIGURE 9: BER performance at macrocell system for joint IA and Alamouti code/joint IA and quasi-orthogonal code/joint IA and Tarokh code.

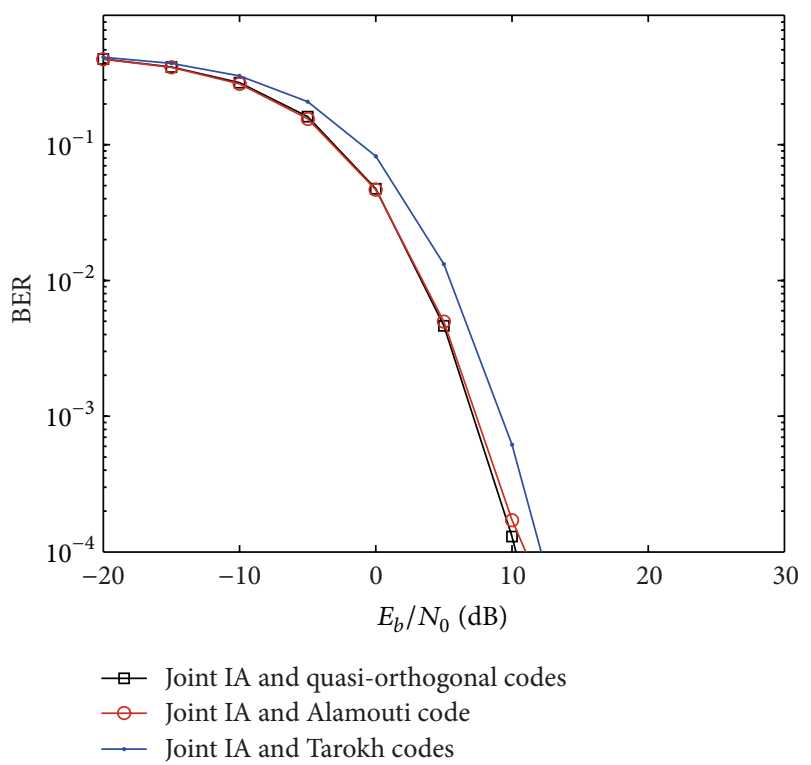

FIGURE 10: BER performance at small-cell system for joint IA and Alamouti code/joint IA and quasi-orthogonal code/joint IA and Tarokh code.

modulation (16-QAM) is used for the joint IA and Tarokh code and therefore it is more prone to errors than the other two SFBC schemes that use QPSK modulation.

\section{Conclusions}

In this paper, we present a general formulation of the diversity-oriented joint IA and SFBC method for the downlink of heterogeneous based systems, where a set of smallcells coexists with a macrocell sharing the same spectrum.
The full-coordinated method provides the best performance but with very high intersystem information exchange requirements. On the other hand, for the uncoordinated-static approach, there is no need for information exchange between the two systems but the macrocell system experiences the worst performance, which is not acceptable for the macrocell system, since it has the priority to access the available resources. To overcome the shortcomings of full-coordinated and the uncoordinated-static methods, we designed the joint IA and SFBC method that can be applied to any SFBC.

The proposed joint IA and SFBC scheme enables the small-cell system to opportunistically access the free space resources of the macrocell system without degrading its performance. Moreover, this method provides improved performance with comparable intersystem information exchange requirements to the uncoordinated-static approach. Thus, the proposed method allows the network to achieve the benefits of full-coordinated and uncoordinated-static methods without their main drawbacks. As one of the requirements of $5 \mathrm{G}$ is to increase spectral efficiency by a factor of about 10, the proposed method will contribute to this goal, and thus it can be very useful for the future $5 \mathrm{G}$ based networks.

\section{Conflict of Interests}

The authors declare that there is no conflict of interests regarding the publication of this paper.

\section{Acknowledgments}

This work was supported by the Portuguese Fundação para a Ciência e Tecnologia (FCT) COPWIN (PTDC/EEI-TEL/ 1417/2012), ADIN (PTDC/EEI-TEL/2990/2012), and HETCOP (UID/EEA/50008/2013) projects and FCT grant for the first (SFRH/BD/94548/2013) and second (SFRH/BPD/95375/2013) authors.

\section{References}

[1] A. Damnjanovic, J. Montojo, Y. Wei et al., "A survey on 3GPP heterogeneous networks," IEEE Wireless Communications, vol. 18, no. 3, pp. 10-21, 2011.

[2] RealWireless, "An assessment of the value of small cell services to operators," 2012, http://www.realwireless.biz/small-cells-asa-service-trials-report/.

[3] J. Hoadley and P. Maveddat, "Enabling small cell deployment with HetNet," IEEE Wireless Communications, vol. 19, no. 2, pp. 4-5, 2012.

[4] T. Zahir, K. Arshad, A. Nakata, and K. Moessner, "Interference management in femtocells," IEEE Communications Surveys and Tutorials, vol. 15, no. 1, pp. 293-311, 2013.

[5] A. Goldsmith, S. A. Jafar, I. Maric, and S. Srinivasa, "Breaking spectrum gridlock with cognitive radios: an information theoretic perspective," Proceedings of the IEEE, vol. 97, no. 5, pp. 894-914, 2009.

[6] Z. Bharucha, H. Haas, G. Auer, and I. Cosovic, "Femto-cell resource partitioning," in Proceedings of the IEEE Global Communications Conference Workshop (GLOBECOM '09), pp. 1-6, IEEE, Honolulu, Hawaii, USA, November-December 2009. 
[7] J. Góra, K. I. Pedersen, A. Szufarska, and S. Strzy, "Cell-specific uplink power control for heterogeneous networks in LTE," in Proceedings of the 72nd Vehicular Technology Conference Fall (VTC 2010-Fall '10), pp. 1-5, IEEE, Ottawa, Canada, September 2010.

[8] E. Yaacoub and Z. Dawy, "Enhancing the performance of OFDMA underlay cognitive radio networks via secondary pattern nulling and primary beam steering," in Proceedings of the IEEE Wireless Communications and Networking Conference (WCNC '11), pp. 1476-1481, Cancun, Mexico, March 2011.

[9] V. R. Cadambe and S. A. Jafar, "Interference alignment and degrees of freedom of the k-user interference channel," IEEE Transactions on Information Theory, vol. 54, no. 8, pp. 34253441, 2008.

[10] C. Suh, M. Ho, and D. N. C. Tse, "Downlink interference alignment," IEEE Transactions on Communications, vol. 59, no. 9, pp. 2616-2626, 2011.

[11] F. Zhao, W. Wang, and H. Chen, "Interference alignment and fairness algorithms for MIMO cognitive radio systems," Mobile Information Systems, vol. 2015, Article ID 907142, 8 pages, 2015.

[12] W. Shin, N. Lee, W. Noh et al., "Hierarchical interference alignment for heterogeneous networks with multiple antennas," in Proceedings of the IEEE International Conference on Communications Workshops (ICC '11), pp. 1-6, IEEE, Kyoto, Japan, June 2011.

[13] B. Guler and A. Yener, "Selective interference alignment for MIMO cognitive femtocell networks," IEEE Journal on Selected Areas in Communications, vol. 32, no. 3, pp. 439-450, 2014.

[14] M. Maso, M. Debbah, and L. Vangelista, "A distributed approach to interference alignment in OFDM-based two-tiered networks," IEEE Transactions on Vehicular Technology, vol. 62, no. 5, pp. 1935-1949, 2013.

[15] K. Huang and R. Zhang, "Cooperative feedback for multiantenna cognitive radio networks," IEEE Transactions on Signal Processing, vol. 59, no. 2, pp. 747-758, 2011.

[16] D. Castanheira, A. Silva, and A. Gameiro, "Set optimization for efficient interference alignment in heterogeneous networks," IEEE Transactions on Wireless Communications, vol. 13, no. 10, pp. 5648-5660, 2014.

[17] S. K. Sharma, S. Chatzinotas, and B. Ottersten, "Interference alignment for spectral coexistence of heterogeneous networks," EURASIP Journal on Wireless Communications and Networking, vol. 2013, no. 1, article 46, 2013.

[18] D. Castanheira, A. Silva, and A. Gameiro, "Null-space cognitive precoding for heterogeneous networks," IET Communications, vol. 8, no. 5, pp. 555-563, 2014.

[19] S. S. Ali, D. Castanheira, A. Silva, and A. Gameiro, "Downlink cognitive interference alignment for heterogeneous networks," in Proceedings of the 21st International Conference on Telecommunications (ICT '14), pp. 236-240, IEEE, Lisbon, Portugal, May 2014.

[20] M. K. Karakayali, G. J. Foschini, and R. A. Valenzuela, "Network coordination for spectrally efficient communications in cellular systems," IEEE Wireless Communications, vol. 13, no. 4, pp. 56$61,2006$.

[21] S. S. Ali, D. Castanheira, A. Silva, and A. Gameiro, "Transmission cooperative strategies for MIMO-OFDM heterogeneous networks," Radioengineering, vol. 24, no. 2, pp. 431-441, 2015.

[22] S. M. Alamouti, "A simple transmit diversity technique for wireless communications," IEEE Journal on Selected Areas in Communications, vol. 16, no. 8, pp. 1451-1458, 1998.
[23] N. Gomes, P. Monteiro, and A. Gameiro, Next Generation Wireless Communications Using Radio over Fiber, John Wiley and Sons, Chichester, UK, 2012.

[24] R. W. Heath Jr. and A. J. Paulraj, "Linear dispersion codes for MIMO systems based on frame theory," IEEE Transactions on Signal Processing, vol. 50, no. 10, pp. 2429-2441, 2002.

[25] B. Hassibi and B. M. Hochwald, "High-rate codes that are linear in space and time," IEEE Transactions on Information Theory, vol. 48, no. 7, pp. 1804-1824, 2002.

[26] O. E. Ayach, S. W. Peters, and R. W. J. Heath, "The practical challenges of interference alignment," IEEE Wireless Communications, vol. 20, no. 1, pp. 35-42, 2013.

[27] O. Tirkkonen, A. Boariu, and A. Hottinen, "Minimal nonorthogonality rate 1 space-time block code for 3+ Tx antennas," in Proceedings of the IEEE 6th International Symposium on Spread Spectrum Techniques and Applications, vol. 2, pp. 429432, Parsippany, NJ, USA, September 2000.

[28] V. Tarokh, N. Seshadri, and A. R. Calderbank, "Space-time codes for high data rate wireless communication: performance criterion and code construction," IEEE Transactions on Information Theory, vol. 44, no. 2, pp. 744-765, 1998. 

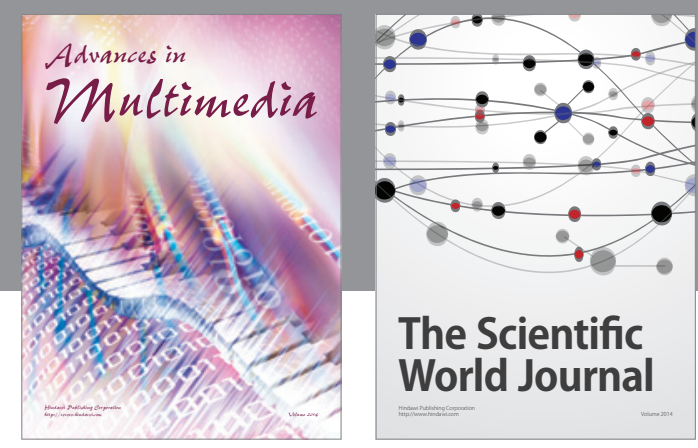

The Scientific World Journal
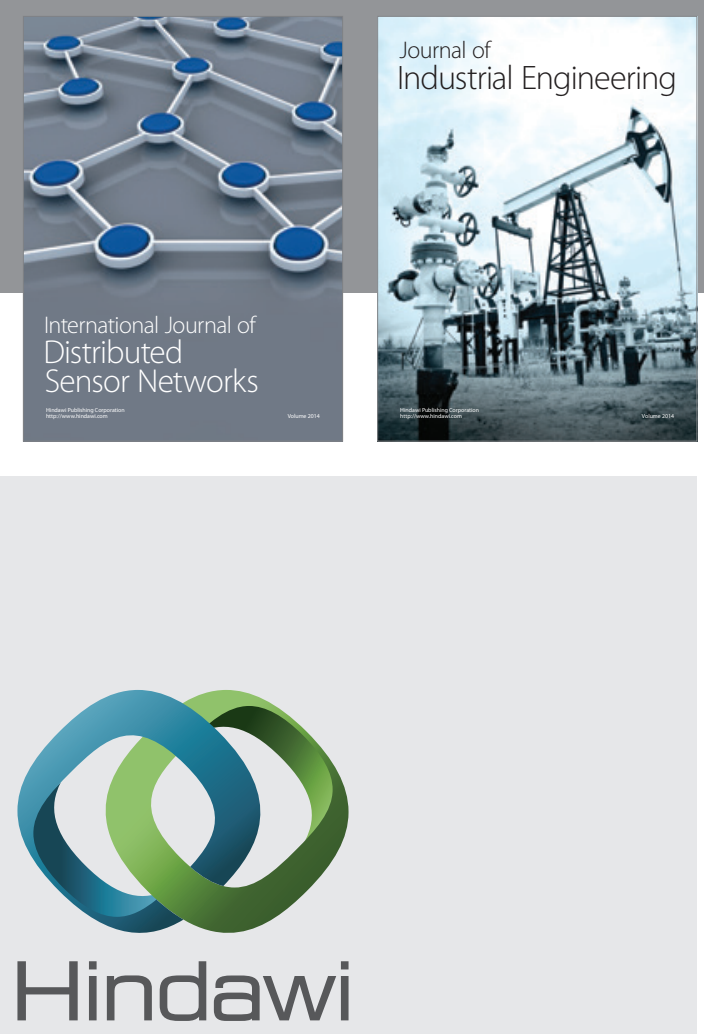

Submit your manuscripts at

http://www.hindawi.com

\section{Computer Networks} and Communications
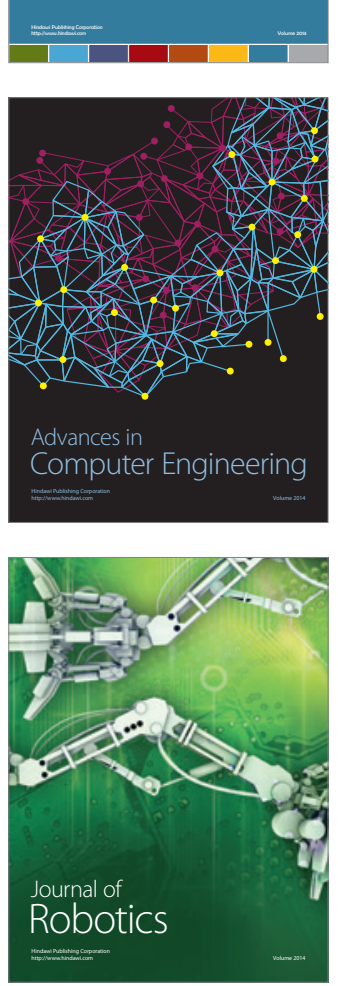
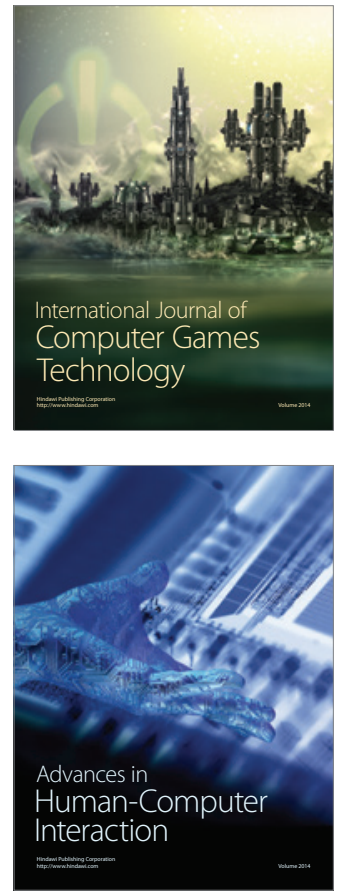
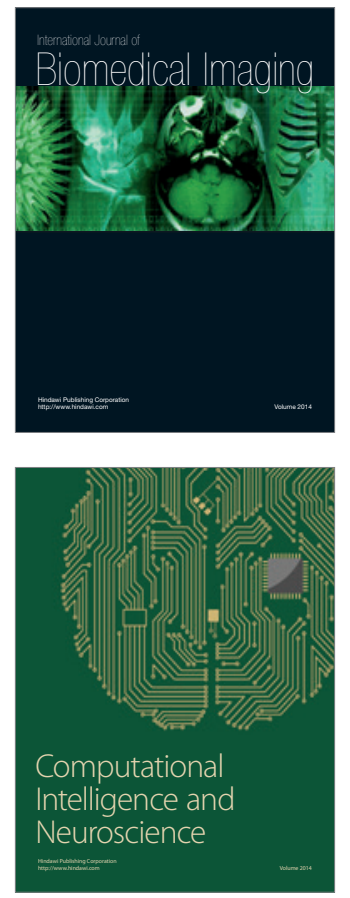
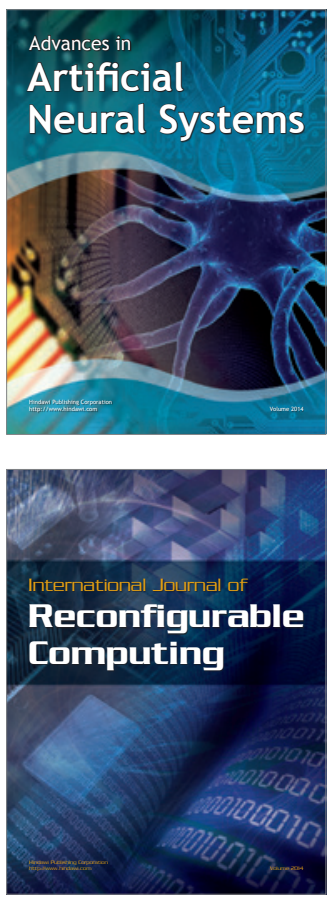
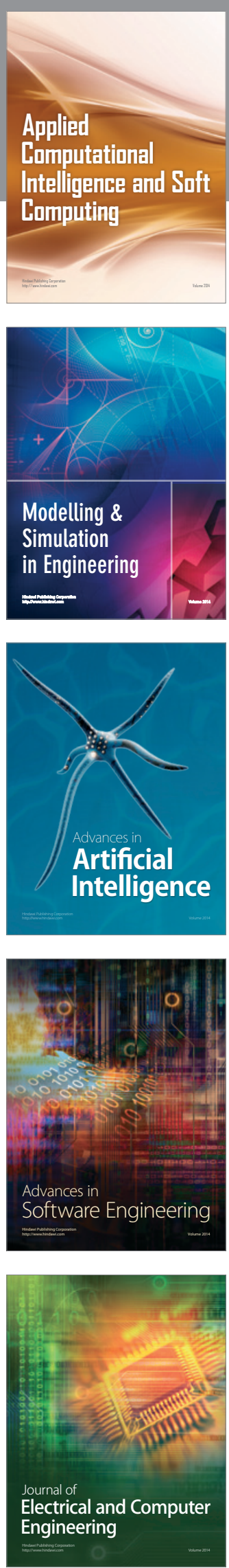